\title{
Clinical implication of Frizzled 2 expression and its association with epithelial-to-mesenchymal transition in hepatocellular carcinoma
}

\author{
TOMONARI ASANO $^{1}$, SUGURU YAMADA ${ }^{1}$, BRYAN C. FUCHS $^{2}$, HIDEKI TAKAMI $^{1}$, MASAMICHI HAYASHI $^{1}$, \\ HIROYUKI SUGIMOTO ${ }^{1}$, TSUTOMU FUJII ${ }^{1}$, KENNETH K. TANABE ${ }^{2}$ and YASUHIRO KODERA ${ }^{1}$ \\ ${ }^{1}$ Department of Gastroenterological Surgery (Surgery II), Nagoya University Graduate School of Medicine, \\ Nagoya, Japan; ${ }^{2}$ Division of Surgical Oncology, Massachusetts General Hospital Cancer Center \\ and Harvard Medical School, Boston, MA, USA
}

Received February 6, 2017; Accepted March 22, 2017

DOI: $10.3892 /$ ijo.2017.3937

\begin{abstract}
The epithelial-to-mesenchymal transition (EMT) is an initial, critical step in hepatocellular carcinoma (HCC) tumor invasion and metastasis. Frizzled $2(\mathrm{Fzd} 2)$ expression might drive EMT through the non-canonical Wnt pathway, one of the various EMT signaling pathways. The expression of epithelial (E-cadherin) and mesenchymal (vimentin) markers, as well as that of Wnt5b, Stat3, IL-6, Jak2 and Fzd2, were measured in 15 HCC cell lines. The EMT status (vimentin to E-cadherin mRNA expression ratio), Fzd 2 mRNA expression, and pSTAT3 protein expression were assessed by immunostaining in $100 \mathrm{HCC}$ patients, and correlations of their expression with clinicopathological factors and prognosis were analyzed. Cell proliferation, migration, and invasiveness were assessed after Fzd2 knockdown. Fzd2 expression was significantly correlated with a mesenchymal phenotype in the HCC cell lines. Treatment of the cell lines with Fzd2 siRNA resulted in significantly reduced migration and invasiveness but did not affect proliferation. A significant correlation was detected between the EMT status and Fzd2 expression in the HCC patients. Multivariate analysis revealed that Fzd 2 expression was an independent predictor of recurrence $(\mathrm{P}=0.034)$.
\end{abstract}

Correspondence to: Dr Suguru Yamada, Department of Gastroenterological Surgery (Surgery II), Nagoya University Graduate School of Medicine, 65 Tsurumai-cho, Showa-ku, Nagoya, Aichi 466-8550, Japan

E-mail: suguru@med.nagoya-u.ac.jp

Abbreviations: EMT, epithelial-to-mesenchymal transition; HCC, hepatocellular carcinoma; Fzd2, Frizzled 2; mRNA, messenger RNA; siRNA, short interfering RNA; HBV, hepatitis B virus; HCV, hepatitis $\mathrm{C}$ virus; well, well-differentiated adenocarcinoma; mod, moderately differentiated adenocarcinoma; poor, poorly differentiated adenocarcinoma; AFP, $\alpha$-fetoprotein

Key words: Frizzled 2, epithelial-to-mesenchymal transition, hepatocellular carcinoma
Patients with high Fzd2 expression had significantly poorer recurrence-free survival than those with low expression $(\mathrm{P}=0.03)$. Finally, pSTAT3 expression was significantly correlated with the EMT and Fzd 2 status $(\mathrm{P}=0.0028$, and $\mathrm{P}=0.0066$, respectively). Fzd2 expression induced EMT and enhanced cell migration and invasiveness, and it might be a novel predictor of HCC recurrence. Furthermore, Stat 3 might be controlled by both the Wnt5/Fzd2 and IL-6/Jak2 signaling pathways and play an important role in EMT.

\section{Introduction}

Hepatocellular carcinoma (HCC) is a common and aggressive human malignancy, and it is currently the second most common cause of cancer-related death worldwide $(1,2)$. Despite great efforts to improve patient outcomes through advances in diagnostic and therapeutic modalities (3), the 5 -year survival of $\mathrm{HCC}$ is still $<50 \%$ (2). Metastasis is the most deadly and least understood aspect of cancer and one of the primary reasons for the high mortality of HCC (4). Increasing evidence indicates that the epithelial-to-mesenchymal transition (EMT), a process by which epithelial cells lose polarity, gain migratory and invasive properties and are converted to a mesenchymal phenotype (5-7), is an initial and critical step in HCC tumor metastasis (8). We have previously reported that EMT is involved in early disease recurrence and is associated with the IL-6 pathway in HCC (9).

EMT contributes to tissue repair and organ fibrosis as well as promotes cancer progression $(10,11)$ and the generation of cells with stem cell-like properties (12). However, a mechanistic understanding of how specific transcription factors induce EMT is still lacking. Thus, uncovering the signaling pathways through which transcription factors regulate EMT is of significant interest, as this information could have broad biological significance. The Wnt proteins are a family of transcription factors that play critical roles in cell proliferation, migration and invasion (13) by binding to and activating one or more of the ten known Frizzled (Fzd) receptors (14). Many previous studies have demonstrated activation of canonical Wnt/ $\beta$-catenin signaling during EMT (15-17). In addition, a 
study assessing pharmacologic and genetic perturbations has revealed that Fzd2 drives EMT and cell migration through a previously unrecognized, non-canonical pathway that involves molecules such as Fyn and Stat3 (13).

In the present study, expression analyses and in vitro functional analyses were conducted to identify the EMT-inducing factors that significantly influence the biological behavior of HCC. Surgical specimens were also obtained from HCC patients to verify the in vitro findings. Furthermore, the role of the non-canonical Wnt pathway in HCC EMT was analyzed in vitro using $\mathrm{HCC}$ cell lines, as well as in vivo.

\section{Materials and methods}

Cell lines and culture. Fifteen human HCC cell lines [HepG2, HuH-7, HuH-2, Hep3B, SNU182, PLC/PRF/5(P5), HLE, SNU398, SNU449, SNU387, HLF, SNU475, HuH-1, Focus, and SK-Hep] were utilized in the present study. Several human hepatoma cell lines (SNU182, SNU398, SNU449, SNU387 and SNU475) were obtained from the American Type Culture Collection (ATCC, Rockville, MD, USA). In addition, the hepatoma cell lines SK-Hep, P5, HepG2, and Hep3B were kindly provided by Barrie Bode (Northern Illinois University, DeKalb, IL, USA), HuH-7 was contributed by Jake Liang (NIDDK, National Institutes of Health, Bethesda, MD, USA), Focus was provided by Jack Wands (Brown University, Providence, RI, USA), and HLE, HLF, HuH-1 and HuH-2 were obtained from Rikon. All cell lines were propagated in RPMI-1640 medium (Life Technologies Corp., Carlsbad, CA, USA) supplemented with $10 \%$ fetal bovine serum (Life Technologies Corp.). Cultures were incubated at $37^{\circ} \mathrm{C} / 5 \% \mathrm{CO}_{2}$.

Patients and specimens. Cancerous tissues and surrounding non-cancerous hepatic parenchyma were obtained from 100 primary HCC patients who underwent resection at Nagoya University Hospital during the period from May 1994 to December 2003. The study was approved by the Ethics Committee, and informed consent was obtained from all patients. The mean follow-up period was $51.3 \pm 40.5$ months.

Real-time polymerase chain reaction. Total RNA isolated from primary HCC tissues and corresponding non-cancerous tissues was used to generate cDNA, which was amplified using PCR primers specific for E-cadherin, vimentin, Fzd2 and GAPDH. PCR amplification consisted of an initial denaturation step at $94^{\circ} \mathrm{C}$ for $5 \mathrm{~min}$, followed by 30 cycles at $94^{\circ} \mathrm{C}$ for $15 \mathrm{sec}, 60^{\circ} \mathrm{C}$ for $15 \mathrm{sec}$ and $72^{\circ} \mathrm{C}$ for $12 \mathrm{sec}$.

RNA expression was determined by real-time quantitative PCR. Real-time detection of the emission intensity of SYBR Green was performed with an ABI PRISM 7000 Sequence Detector (Perkin-Elmer Applied Biosystems, Foster City, CA, USA). Quantitative PCR was performed at least three times for each sample, and a no-template negative control was used.

The EMT status of each patient's tumor was determined from E-cadherin and vimentin mRNA expression as follows: vimentin/E-cadherin $<2$ = epithelial (E); and vimentin/ E-cadherin $\geq 2=$ mesenchymal $(\mathrm{M})$.

Fzd2 expression in each patient's tumor was classified according to the mRNA expression in cancerous tissue versus that in non-cancerous tissue as follows: Fzd2 expression in cancerous tissue/Fzd2 expression in non-cancerous tissue $<2=$ Fzd2 low-expression group; and Fzd2 expression in cancerous tissue/Fzd2 expression in non-cancerous tissue $\geq 2=$ Fzd2 high-expression group.

Western blot analysis. Cell lysates were prepared and loaded onto $4-12 \%$ gels to separate proteins by SDS-PAGE. The proteins were then transferred to polyvinylidene difluoride membranes. The membranes were blocked with phosphatebuffered saline-Tween containing $5 \%(w / v)$ non-fat milk for $2 \mathrm{~h}$ at room temperature with shaking and then incubated overnight at $4^{\circ} \mathrm{C}$ with a primary antibody (rat anti-Frizzled2 diluted 1:1,000; R\&S Systems, \#MAB1307).

$F z d 2$ short interfering RNA transfection. HLF cells were seeded in 6-well plates $\left(2 \times 10^{5}\right.$ cells/well) and transfected the next day with either $30 \mathrm{nM}$ predesigned short interfering RNA (siRNA) targeting Fzd2 or control siRNA (GE Healthcare, Buckinghamshire, UK). After $72 \mathrm{~h}$, the Fzd2 protein and mRNA levels were analyzed by western blotting and real-time PCR, respectively.

Cell proliferation, migration, and invasion assays. HLF cells were seeded in 96-well plates $\left(5 \times 10^{3}\right.$ cells/well) and transfected the following day with Fzd2 or control siRNA. Cell proliferation was evaluated using premix WST-1 reagent (Takara Bio, Japan), and absorbance was measured at $440 \mathrm{~nm}$.

Migratory ability was assessed by wound healing assays, which were performed using the culture insert method (Ibidi GmbH, Germany). When the cell layer was confluent at $24 \mathrm{~h}$ after transfection, the culture insert was removed. The area of migration was measured using ImageJ software (Wayne Rasband, National Institute of Health, USA) at $24 \mathrm{~h}$ after removal of the insert.

Cell invasion was assessed using Matrigel invasion chambers. HLF cells were seeded into 6 -well plates $\left(5 \times 10^{4}\right.$ cells/well) and transfected the following day with Fzd2 or control siRNA. At $48 \mathrm{~h}$ post-transfection, the cells were plated in transwell chambers (BD Bioscience) pre-coated with Matrigel Invasion Chamber medium. After approximately $24 \mathrm{~h}$, non-invading cells were removed, and invasive cells attached to the lower surface of the membrane were stained. The number of invasive cells was determined from five randomly selected fields of view.

Statistical analysis. Between-group differences were evaluated using Fisher's exact test or the $\chi^{2}$ test. In addition, the recurrence-free and overall survival rates were calculated using the Kaplan-Meier method, and differences between survival curves were analyzed using the log-rank test. Independent prognostic factors were analyzed using Cox proportional hazards regression models. The data are presented as the mean $\pm \mathrm{SD}$, and a P-value of $<0.05$ was considered statistically significant. Analysis was conducted using JMP version 11 software (JMP, SAS Institute, Cary, NC, USA).

\section{Results}

E-cadherin, vimentin and EMT-inducing factors in human HCC cell lines. E-cadherin and vimentin mRNA expression 

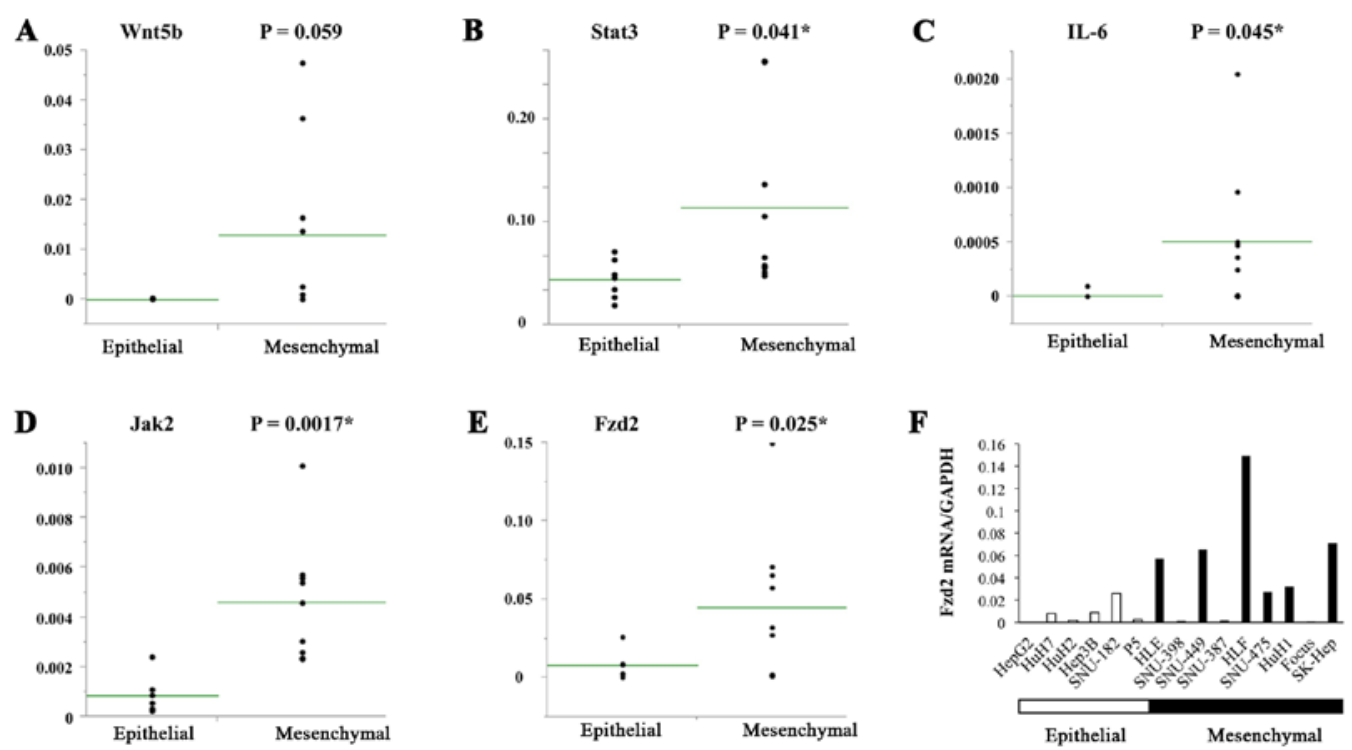

Figure 1. Correlations between the mRNA expression of EMT-inducing transcription factors and the EMT status. mRNA expression of the relevant transcription factors (A) Wnt5b, (B) Stat3, (C) IL-6, (D) Jak2 and (E) Fzd2 were measured by real-time PCR in 15 HCC cell lines. Fzd2 mRNA expression in the cell lines is shown (F). mRNA, messenger RNA; EMT, epithelial-to-mesenchymal transition; and HCC, hepatocellular carcinoma. A single asterisk indicates statistical significance.
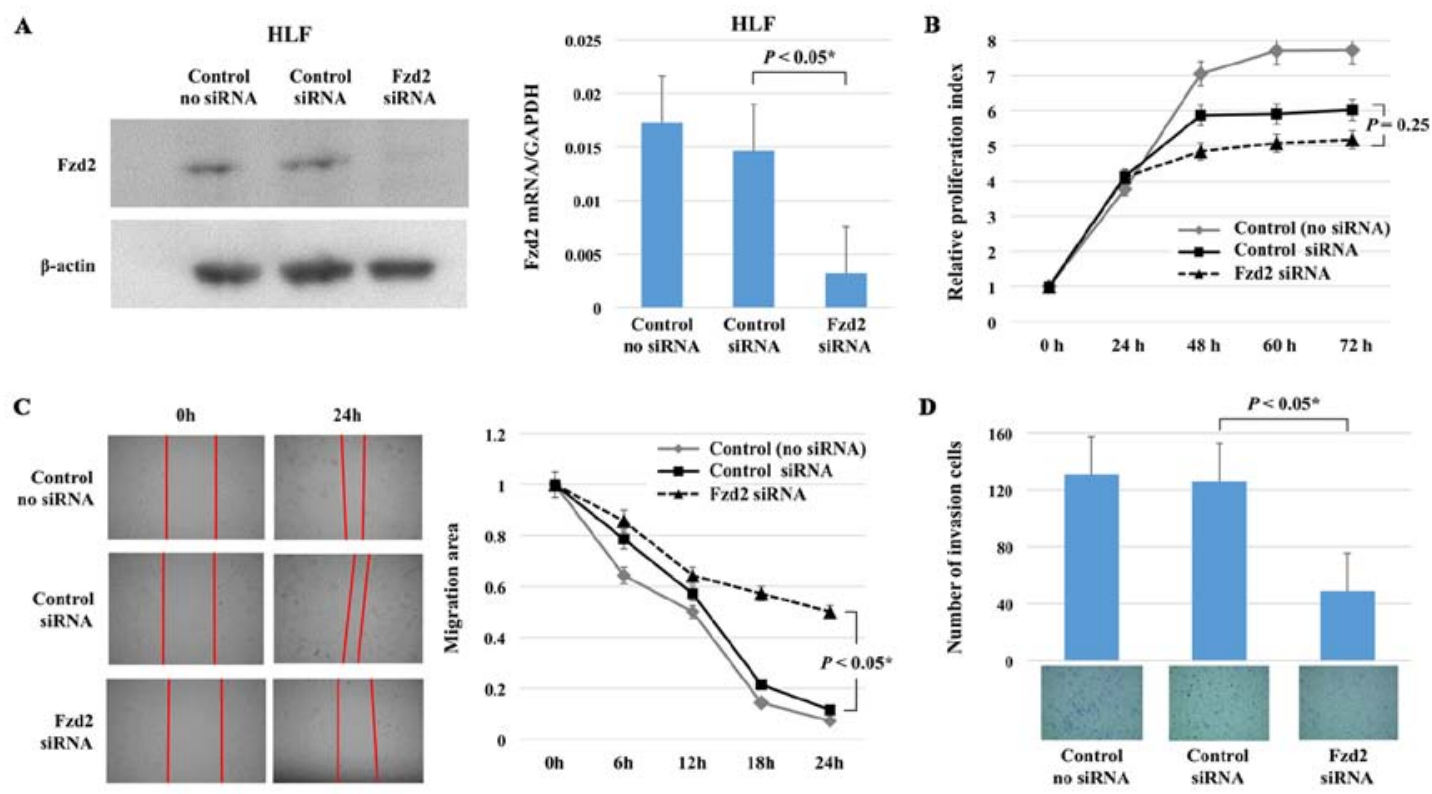

Figure 2. Knockdown of Fzd2 and functional analysis. (A) Fzd2 protein and mRNA expression was decreased by transfection of cells with Fzd2 siRNA. (B) HLF cells transfected with Fzd2 siRNA did not show significantly reduced cell proliferation compared with that of the controls. (C) HLF cells treated with Fzd2 siRNA exhibited reduced migration compared with that of the controls. (D) HLF cells transfected with Fzd2 siRNA showed significantly reduced invasiveness compared with that of the controls. A single asterisk indicates statistical significance.

was measured by real-time PCR in $15 \mathrm{HCC}$ cell lines to determine the extent of EMT. Six of the cell lines (HepG2, HuH7, HuH2, Hep3B, SNU-182, and P5) were classified as epithelial, and nine (HLE, SNU-398, SNU-449, SNU-387, HLF, SNU-475, HuH1, Focus, and SK-Hep) were classified as mesenchymal based on their E-cadherin and vimentin expression.

The levels of EMT-inducing factors (Wnt5b, Stat3, IL-6, Jak2, and Fzd2) were also measured in the HCC cell lines, and their associations with EMT were examined. Several of these factors (Stat3, IL-6, Jak2, and Fzd2) were expressed at high levels in the mesenchymal HCC cell lines (Fig. 1A-E).
In particular, Fzd 2 was highly expressed in 4 of the 9 mesenchymal cell lines (HLF, SK-Hep, SNU-449 and HLE), but it was not expressed in any of the 6 epithelial cell lines (Fig. 1F).

Impact of $F z d 2$ expression on proliferation, migration and invasion. To further verify the relationship of Fzd2 with EMT in HCC, Fzd2 siRNA was transfected into mesenchymal HLF cells, and the impacts on cell proliferation, migration, and invasion were evaluated. Fzd 2 mRNA and protein expression was significantly inhibited in the HLF cells following treatment with Fzd2 siRNA (Fig. 2A). 
Table I. Correlation between Fzd2 expression and clinicopathological features.

\begin{tabular}{|c|c|c|c|}
\hline Characteristic & Fzd2 low $(n=64)$ & Fzd2 high $(n=36)$ & P-value \\
\hline Age $(>65 / \leq 64)$ & $32 / 32$ & $20 / 16$ & 0.74 \\
\hline Male/female & $51 / 13$ & $32 / 4$ & 0.25 \\
\hline Virus (HBV/HCV/others) & $9 / 42 / 13$ & $6 / 23 / 7$ & 0.53 \\
\hline \multicolumn{4}{|l|}{ Histologic type of tumor } \\
\hline Mod/Well/Poor/others & $43 / 7 / 4 / 10$ & $24 / 6 / 0 / 6$ & 0.41 \\
\hline \multicolumn{4}{|l|}{ Tumor size $(\mathrm{cm})$} \\
\hline$>3 / \leq 3$ & $38 / 26$ & $23 / 13$ & 0.48 \\
\hline \multicolumn{4}{|l|}{ Tumor multiplicity } \\
\hline Solitary/multiple & $50 / 14$ & $22 / 13$ & 0.14 \\
\hline \multicolumn{4}{|l|}{ Pattern of tumor growth } \\
\hline Expansive/infiltrative & $53 / 11$ & $30 / 6$ & 0.60 \\
\hline \multicolumn{4}{|l|}{ Formation of fibrous capsule } \\
\hline Present/absent & $48 / 16$ & $20 / 16$ & 0.23 \\
\hline \multicolumn{4}{|l|}{ Septal formation } \\
\hline Present/absent & $44 / 20$ & $20 / 16$ & 0.36 \\
\hline \multicolumn{4}{|l|}{ Vascular invasion } \\
\hline Present/absent & $15 / 49$ & $12 / 24$ & 0.46 \\
\hline \multicolumn{4}{|l|}{ AFP level (ng/ml) } \\
\hline$>20 / \leq 20$ & $37 / 27$ & $20 / 16$ & 0.98 \\
\hline \multicolumn{4}{|l|}{ Pugh-Child's classification } \\
\hline $\mathrm{A} / \mathrm{B}$ & $58 / 6$ & $34 / 1$ & 0.62 \\
\hline \multicolumn{4}{|l|}{ Pathological stage } \\
\hline III/III IVa & $42 / 22$ & $19 / 17$ & 0.31 \\
\hline \multicolumn{4}{|l|}{ EMT status } \\
\hline Epithelial/mesenchymal & $51 / 13$ & $10 / 26$ & $<0.0001$ \\
\hline
\end{tabular}

$\mathrm{HBV}$, hepatitis B virus; $\mathrm{HCV}$, hepatitis C virus; well, well-differentiated adenocarcinoma; mod, moderately differentiated adenocarcinoma; poor, poorly differentiated adenocarcinoma; AFP, $\alpha$-fetoprotein; EMT, epithelial to mesenchymal transition.

Next, the proliferation, migration and invasiveness of HLF cells treated with Fzd2 siRNA were examined, and the results were compared with those for HLF cells treated with control siRNA. Cell proliferation was not affected by Fzd2 inhibition; however, cell migration and invasiveness were significantly reduced (Fig. 2B-D). These results demonstrated that although Fzd2 knockdown failed to reduce proliferation, it inhibited migration and invasion, indicative of reduced EMT.

Clinical implicationofFzd2 status in HCCpatients. Subsequently, we measured Fzd2 mRNA expression in cancerous and surrounding non-cancerous tissues from $100 \mathrm{HCC}$ patients using real-time PCR. The Fzd2 status was then determined based on the cancerous tissue to non-cancerous tissue ratio, as described in Materials and methods. The patients were classified into an Fzd2 low-expression group (64 patients) or Fzd2 high-expression group (36 patients). Similarly, E-cadherin and vimentin expression was measured by real-time PCR in the cancerous and surrounding non-cancerous tissues from the same patients.
The EMT status was then determined based on the vimentin to E-cadherin ratio (V/E ratio), and the patients were classified into an epithelial group (61 patients) or mesenchymal group (39 patients).

Examination of the relationship between Fzd2 and EMT revealed that Fzd2 expression was significantly higher in the mesenchymal group than in the epithelial group $(\mathrm{P}<0.0001)$ (Fig. 3A). Additionally, a significant correlation was detected between the V/E ratio and Fzd2 expression $(r=0.37, \mathrm{P}<0.0001)$ (Fig. 3B). However, no significant correlation was observed between the Fzd2 status and any other clinicopathological parameter (Table I). These results further indicated that Fzd2 expression was an essential mediator of EMT in HCC.

Prognosis of HCC patients according to the status of EMT and $F z d 2$. Analysis of survival based on the EMT status revealed no difference in overall survival $(\mathrm{P}=0.89)$ but a significant difference in recurrence-free survival between the epithelial and mesenchymal groups $(\mathrm{P}=0.023)$, indicating that 
$\mathbf{A}$

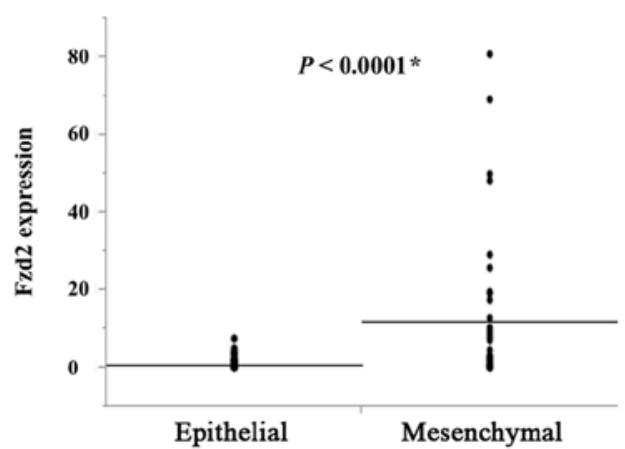

C

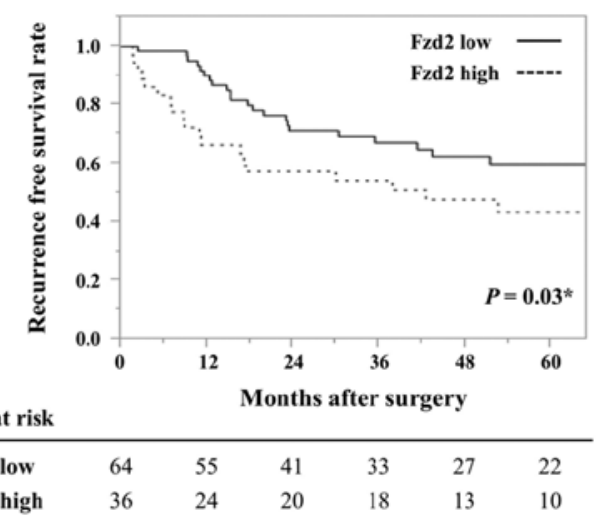

B

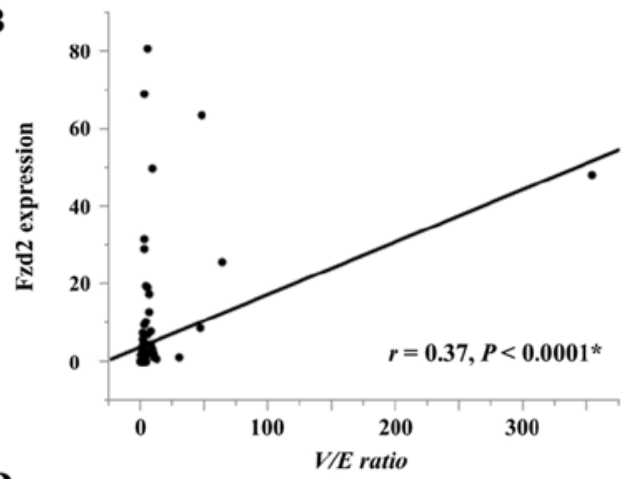

D

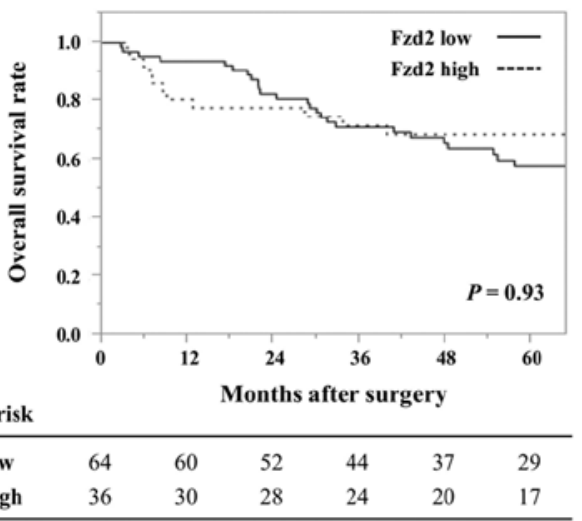

Figure 3. High Fzd2 expression correlated with a mesenchymal phenotype in $100 \mathrm{HCC}$ patients. Fzd2 expression was measured by real-time PCR and analyzed in relation to the EMT status (A) and V/E ratio (B) in 100 HCC patients. Recurrence-free survival (C) and overall survival (D) were analyzed based on Fzd2 expression. EMT, epithelial-to-mesenchymal transition; and $\mathrm{HCC}$, hepatocellular carcinoma. A single asterisk indicates statistical significance.

$\mathbf{A}$

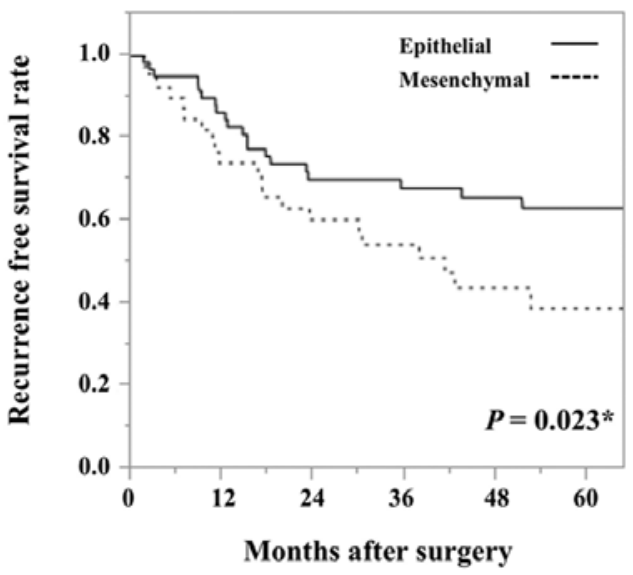

No. at risk

\begin{tabular}{lcccccc}
\hline Epithelial & 61 & 50 & 38 & 33 & 30 & 24 \\
Mesenchymal & 39 & 29 & 23 & 18 & 10 & 8 \\
\hline
\end{tabular}

B

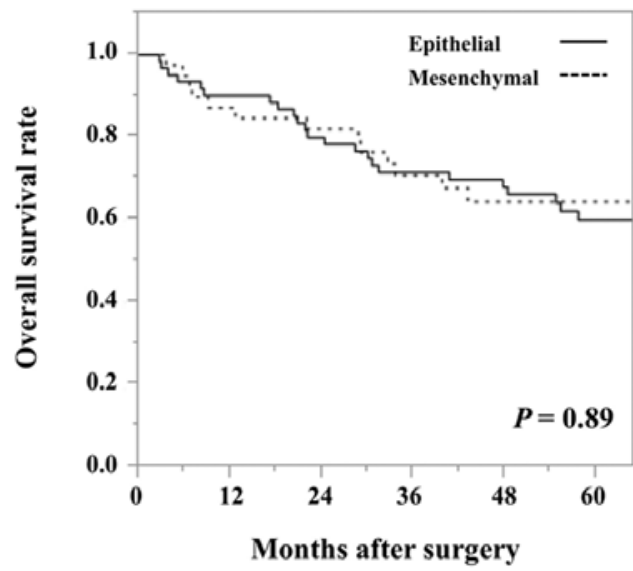

\begin{tabular}{lllllll} 
No. at risk & \multicolumn{10}{l}{} \\
\hline Epithelial & 61 & 55 & 48 & 43 & 39 & 30 \\
Mesenchymal & 39 & 35 & 32 & 25 & 18 & 16 \\
\hline
\end{tabular}

Figure 4. E-cadherin and vimentin mRNA expression measured by real-time PCR in 100 HCC patients, and the EMT status determined based on the vimentin to E-cadherin ratio. Recurrence-free survival (A) and overall survival (B) were analyzed based on the EMT status. A single asterisk indicates statistical significance.

the patients with a mesenchymal tumor were more prone to experiencing earlier recurrence than those with an epithelial tumor (Fig. 4).

Similarly, analysis of survival based on the Fzd2 status revealed a significant difference in recurrence-free survival $(\mathrm{P}=0.03)$ (Fig. 3C) but no difference in overall survival $(\mathrm{P}=0.93)$ (Fig. 3D). Thus, the HCC patients in the Fzd2 high-expression group more frequently experienced earlier recurrence than those in the Fzd2 low-expression group.

Univariate and multivariate analyses of clinicopathological factors for HCC recurrence. Clinicopathological factors were also analyzed as predictive factors for recurrence. Univariate analysis showed that male gender was associated with early 
Table II. Univariate and multivariate analysis for predictors of recurrence-free survival.

\begin{tabular}{|c|c|c|c|c|}
\hline \multirow[b]{2}{*}{ Predictors } & \multicolumn{2}{|c|}{ Univariate analysis } & \multicolumn{2}{|c|}{ Multivariate analysis } \\
\hline & $\mathrm{HR}(95 \% \mathrm{CI})$ & P-value & $\mathrm{HR}(95 \% \mathrm{CI})$ & P-value \\
\hline Age $(\geq 65$ vs $<64)$ & $0.74(0.40-1.32)$ & 0.31 & & \\
\hline Gender (male vs female) & $2.22(0.96-6.46)$ & 0.064 & $2.15(0.92-6.27)$ & 0.077 \\
\hline Tumor size $(3 \mathrm{~cm})$ & $1.43(0.76-2.87)$ & 0.28 & & \\
\hline Histological type & $1.69(0.40-4.74)$ & 0.42 & & \\
\hline Tumor multiplicity & $1.57(0.80-2.97)$ & 0.18 & & \\
\hline Pattern of tumor growth & $1.37(0.58-2.85)$ & 0.44 & & \\
\hline Formation of fibrous capsule & $0.94(0.47-2.02)$ & 0.87 & & \\
\hline Septal formation & $1.53(0.76-3.41)$ & 0.24 & & \\
\hline Vascular invasion & $1.74(0.88-3.38)$ & 0.13 & & \\
\hline AFP & $1.14(0.61-2.19)$ & 0.68 & & \\
\hline Child-Pugh & $0.58(0.094-1.90)$ & 0.42 & & \\
\hline Pathological stage & $1.30(0.68-2.43)$ & 0.42 & & \\
\hline Fzd2 status (high) & $1.89(1.04-3.38)$ & $0.036^{\mathrm{a}}$ & $1.85(1.02-3.31)$ & $0.043^{\mathrm{a}}$ \\
\hline
\end{tabular}

HR, hazard ratio; CI, confidence interval; EMT, epithelial mesenchymal transition; AFP, $\alpha$-fetoprotein; PIVKA II; protein induced by vitamin K

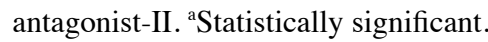

$\mathbf{A}$
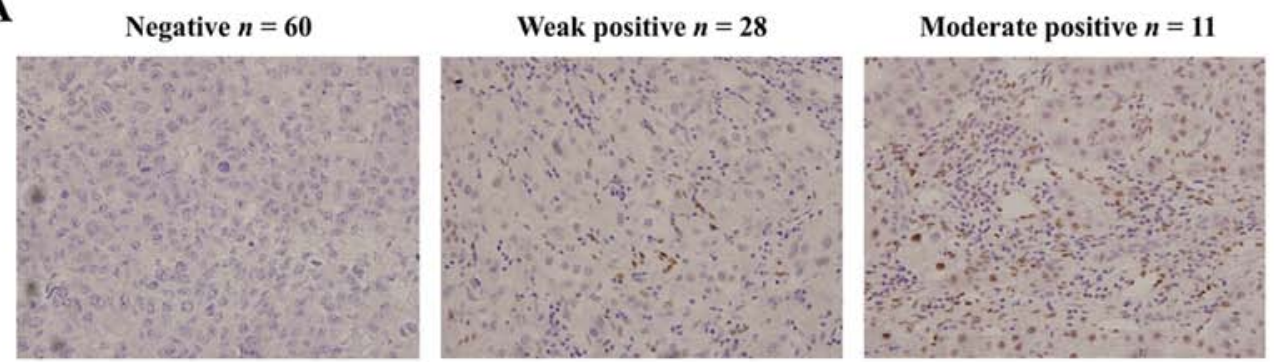

B

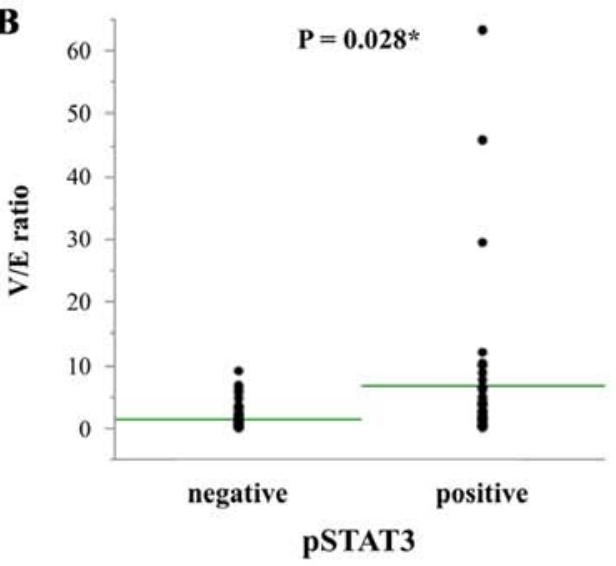

C

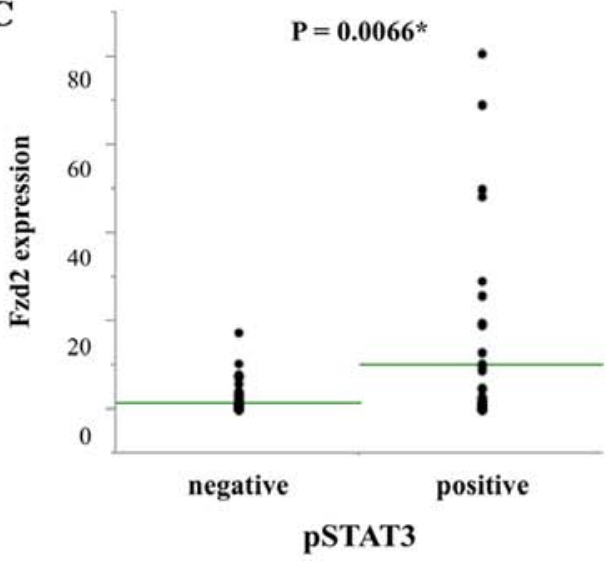

Figure 5. Representative images of Stat 3 expression in HCC patients. Stat 3 was localized to the nuclei of HCC cells (A). Stat 3 expression was correlated with the $\mathrm{V} / \mathrm{E}$ ratio (B) and Fzd2 expression (C). HCC, hepatocellular carcinoma; and V/E ratio, vimentin to E-cadherin ratio. A single asterisk indicates statistical significance.

recurrence, and this association was significant for the patients with high Fzd2 expression $(\mathrm{P}=0.036)$. Multivariate analysis also revealed that high Fzd 2 expression was more strongly associated with earlier recurrence than low Fzd2 expression $(\mathrm{P}=0.043)$ (Table II).
Immunohistochemical analysis of pSTAT3 and the correlations of its expression with the status of EMT and Fzd2 in HCC patients. Immunohistochemical staining for pSTAT3 revealed that it was localized to the nuclei of HCC cells. Negative pSTAT3 staining was observed in 60 out of 100 patients, 
whereas weak, moderate and strong positive staining were observed in 28, 11 and 1 patients, respectively (Fig. 5A). The patients with positive pSTAT3 staining had a significantly higher $\mathrm{V} / \mathrm{E}$ ratio than those with negative staining $(\mathrm{P}=0.0028)$ (Fig. 5B). In addition, they had significantly higher Fzd2 expression $(\mathrm{P}=0.0066)$ (Fig. $5 \mathrm{C})$. These results revealed that pSTAT3 expression was significantly correlated with EMT and the Fzd2 status.

\section{Discussion}

The invasiveness and metastasis of many cancers, including $\mathrm{HCC}$, represent major obstacles for cancer treatment and are associated with poor survival. In this regard, EMT is thought to be an important biological process that plays critical roles in tumor cell invasion and metastasis, and it has been actively investigated in association with HCC (18-20). Thus, studies examining the mechanism underlying EMT and identifying novel targets for controlling HCC invasiveness and metastasis are urgently needed.

Several growth factors, including TGF $\beta$, Wnt, EGF, and HGF, have been shown to trigger EMT both during embryonic development and in normal and transformed cell lines (10). In particular, Wnt5 ligands have been reported to be overexpressed in mesenchymal cells derived from late-stage HCC tumors. Furthermore, Fzd2 has been shown to be overexpressed in late-stage HCC and lung cancer, and its overexpression has been reported to be correlated with poor patient survival (13). In the present study, the correlation among Fzd2 expression, EMT, and patient prognosis was investigated in resected HCC clinical samples. The results revealed that high Fzd 2 expression was strongly correlated with mesenchymal-type tumors among the HCC patients. In addition, the status of EMT and Fzd2 was significantly associated with recurrence-free survival. These data further support the notion that Fzd2 has important roles in the process of EMT. Thus, Fzd2 was demonstrated to play an important role in inducing EMT in HCC, and it could be a novel predictor of early recurrence after $\mathrm{HCC}$ resection.

In recent years, constitutive activation of the Stat 3 signaling pathway has been detected in a variety of human tumors, and Stat 3 activation has frequently been reported in association with tumor invasiveness and metastasis (21-23). In our study, pStat 3 expression was detected in $\leq 40 \%$ of the surgically resected HCC specimens by immunohistochemical analysis, and its expression was strongly correlated with the status of Fzd 2 and EMT. These findings suggest that Stat 3 phosphorylation is involved in the Fzd2-dependent EMT and cell migration. With regard to cell proliferation, migration, and invasiveness in HCC, Fzd2 knockdown or treatment with an anti-Fzd 2 antibody has been reported to result in reduced cell migration and invasion and inhibition of tumor growth and metastasis in a mouse xenograft model (13). In addition, shRNA-Fzd 2 has been shown to suppress cell proliferation in HLF and PLC/PRF/5 cells (24). However, our results showed that the migration and invasiveness of HLF cells were significantly inhibited by treatment with Fzd 2 siRNA, whereas proliferation was only marginally affected. Taken together, these results indicate that the signaling pathway regulating cell proliferation might also be influenced by mechanisms independent of Wnt5/Fzd2.
EMT is known to generate cells with properties of stem cells (12). We have previously reported that the IL-6 pathway is associated with HCC EMT (9), and recent reports have demonstrated that IL- 6 promotes Stat 3 activation and expression in stem cells in HCC $(25,26)$. Interestingly, Janus kinase, which also activates Stat3, is required for IL-6- but not for Wnt5/Fzd2-mediated activation of Stat3 (13). In general, Stat3 is known to function downstream of both Wnt5/Fzd2 and the IL-6/Jak2 signaling pathway. Although additional studies are needed to clarify these findings, we hypothesize that Wnt5/ Fzd 2 might be more closely associated with migration and invasion, whereas IL-6/Jak2 might regulate stemness and proliferation. In any case, at least two pathways are involved in the process of EMT, and combined treatment with an antiFzd2 antibody and IL-6R antibody might more effectively suppress Stat3 activation than the blockade of either pathway alone in the treatment of HCC (27).

In conclusion, Fzd2 regulates EMT and cell migration and invasion in HCC, and it might be a novel predictor of recurrence in HCC. Furthermore, Stat 3 might be controlled by both Wnt5/Fzd2 and the IL-6/Jak2 signaling pathway and play an important role in EMT.

\section{References}

1. Poon D, Anderson BO, Chen LT, Tanaka K, Lau WY, Van Cutsem E, Singh H, Chow WC, Ooi LL, Chow P, et al; Asian Oncology Summit: Management of hepatocellular carcinoma in Asia: Consensus statement from the Asian Oncology Summit 2009. Lancet Oncol 10: 1111-1118, 2009.

2. McGlynn KA, Petrick JL and London WT: Global epidemiology of hepatocellular carcinoma: An emphasis on demographic and regional variability. Clin Liver Dis 19: 223-238, 2015.

3. Thorgeirsson SS and Grisham JW: Molecular pathogenesis of human hepatocellular carcinoma. Nat Genet 31: 339-346, 2002.

4. Chaffer CL and Weinberg RA: A perspective on cancer cell metastasis. Science 331: 1559-1564, 2011.

5. Thompson EW, Newgreen DF and Tarin D: Carcinoma invasion and metastasis: A role for epithelial-mesenchymal transition? Cancer Res 65: 5991-5995, discussion 5995, 2005.

6. Thiery JP: Epithelial-mesenchymal transitions in tumour progression. Nat Rev Cancer 2: 442-454, 2002.

7. Thiery JP and Sleeman JP: Complex networks orchestrate epithelial-mesenchymal transitions. Nat Rev Mol Cell Biol 7: 131-142, 2006.

8. van Zijl F, Krupitza G and Mikulits W: Initial steps of metastasis: Cell invasion and endothelial transmigration. Mutat Res 728: 23-34, 2011.

9. Yamada S, Okumura N, Wei L, Fuchs BC, Fujii T, Sugimoto H, Nomoto S, Takeda S, Tanabe KK and Kodera Y: Epithelial to mesenchymal transition is associated with shorter disease-free survival in hepatocellular carcinoma. Ann Surg Oncol 21: 3882-3890, 2014.

10. Thiery JP, Acloque H, Huang RY and Nieto MA: Epithelialmesenchymal transitions in development and disease. Cell 139: 871-890, 2009.

11. Micalizzi DS, Farabaugh SM and Ford HL: Epithelialmesenchymal transition in cancer: Parallels between normal development and tumor progression. J Mammary Gland Biol Neoplasia 15: 117-134, 2010.

12. Mani SA, Guo W, Liao MJ, Eaton EN, Ayyanan A, Zhou AY, Brooks M, Reinhard F, Zhang CC, Shipitsin M, et al: The epithelial-mesenchymal transition generates cells with properties of stem cells. Cell 133: 704-715, 2008

13. Gujral TS, Chan M, Peshkin L, Sorger PK, Kirschner MW and MacBeath G: A noncanonical Frizzled2 pathway regulates epithelial-mesenchymal transition and metastasis. Cell 159: 844-856, 2014.

14. Willert K, Brown JD, Danenberg E, Duncan AW, Weissman IL, Reya T, Yates JR III and Nusse R: Wnt proteins are lipid-modified and can act as stem cell growth factors. Nature 423: 448-452, 2003. 
15. Deka J, Wiedemann N, Anderle P, Murphy-Seiler F, Bultinck J, Eyckerman S, Stehle JC, André S, Vilain N, Zilian O, et al: Bcl9/Bcl9l are critical for Wnt-mediated regulation of stem cell traits in colon epithelium and adenocarcinomas. Cancer Res 70: 6619-6628, 2010.

16. Gupta S, Iljin K, Sara H, Mpindi JP, Mirtti T, Vainio P, Rantala J, Alanen K, Nees M and Kallioniemi O: FZD4 as a mediator of ERG oncogene-induced WNT signaling and epithelial-tomesenchymal transition in human prostate cancer cells. Cancer Res 70: 6735-6745, 2010.

17. Wu ZQ, Brabletz T, Fearon E, Willis AL, Hu CY, Li XY and Weiss SJ: Canonical Wnt suppressor, Axin2, promotes colon carcinoma oncogenic activity. Proc Natl Acad Sci USA 109: 11312-11317, 2012

18. Lee TK, Poon RT, Yuen AP, Ling MT, Kwok WK, Wang XH, Wong YC, Guan XY, Man K, Chau KL, et al: Twist overexpression correlates with hepatocellular carcinoma metastasis through induction of epithelial-mesenchymal transition. Clin Cancer Res 12: 5369-5376, 2006.

19. Giannelli G, Bergamini C, Fransvea E, Sgarra C and Antonaci S: Laminin-5 with transforming growth factor- $\beta 1$ induces epithelial to mesenchymal transition in hepatocellular carcinoma. Gastroenterology 129: 1375-1383, 2005.

20. Lee TK, Man K, Poon RT, Lo CM, Yuen AP, Ng IO, Ng KT, Leonard W and Fan ST: Signal transducers and activators of transcription $5 \mathrm{~b}$ activation enhances hepatocellular carcinoma aggressiveness through induction of epithelial-mesenchymal transition. Cancer Res 66: 9948-9956, 2006.
21. Chen CL, Hsieh FC, Lieblein JC, Brown J, Chan C, Wallace JA, Cheng G, Hall BM and Lin J: Stat 3 activation in human endometrial and cervical cancers. Br J Cancer 96: 591-599, 2007.

22. Kusaba T, Nakayama T, Yamazumi K, Yakata Y, Yoshizaki A, Nagayasu T and Sekine I: Expression of p-STAT3 in human colorectal adenocarcinoma and adenoma; correlation with clinicopathological factors. J Clin Pathol 58: 833-838, 2005.

23. Suiqing C, Min Z and Lirong C: Overexpression of phosphorylated-STAT3 correlated with the invasion and metastasis of cutaneous squamous cell carcinoma. J Dermatol 32: 354-360, 2005.

24. Tomizawa M, Shinozaki F, Motoyoshi Y, Sugiyama T, Yamamoto S and Ishige N: Suppression of hepatocellular carcinoma cell proliferation by short hairpin RNA of frizzled 2 with Sonazoidenhanced irradiation. Int J Oncol 48: 123-129, 2016.

25. Won C, Kim BH, Yi EH, Choi KJ, Kim EK, Jeong JM, Lee JH, Jang JJ, Yoon JH, Jeong WI, et al: Signal transducer and activator of transcription 3-mediated CD133 up-regulation contributes to promotion of hepatocellular carcinoma. Hepatology 62: 1160-1173, 2015

26. Wan S, Zhao E, Kryczek I, Vatan L, Sadovskaya A, Ludema G, Simeone DM, Zou W and Welling TH: Tumor-associated macrophages produce interleukin 6 and signal via STAT3 to promote expansion of human hepatocellular carcinoma stem cells. Gastroenterology 147: 1393-1404, 2014.

27. Ghoshal S, Fuchs BC and Tanabe KK: STAT3 is a key transcriptional regulator of cancer stem cell marker CD133 in HCC. Hepatobiliary Surg Nutr 5: 201-203, 2016. 